\title{
RF Characterization of Substations: Parameters for Impulsive Noise Models based on the Equipment Voltage
}

\author{
Fabien Sacuto \\ McGill University \\ Montreal, Canada \\ Email: \\ fabien.sacuto@mail.mcgill.ca
}

\author{
Fabrice Labeau \\ McGill University \\ Montreal, Canada \\ Email: \\ fabrice.labeau@mcgill.ca
}

\author{
Basile.L Agba \\ IREQ \\ Varennes, Canada \\ Email: \\ agba.basileL@ireq.ca
}

\begin{abstract}
Installing wireless Intelligent Electronic Devices (IED) for Substation Automation (SA) requires a thorough study of the electromagnetic radiations coming from the power equipment. In our previous work, we have performed a measurement campaign within several substations working under different voltages and we have recorded around 120 sequences of impulsive noise samples in the $700 \mathrm{MHz}-2.5 \mathrm{GHz}$ band. In this paper, we present a method to classify substation impulsive noise in order to characterize a representative Radio Frequency (RF) environment of substations for specific substation voltages. The main contribution of this work is to provide representative impulsive noise characteristics in order to calculate parameters for impulsive noise models and to improve the characterization of substation RF noise. To reach this objective, we classify impulsive noise characteristics, such as the impulse amplitude, the impulse duration and the repetition rate for substations under $25 \mathrm{kv}$, $230 \mathrm{kV}, 315 \mathrm{kV}$ and $735 \mathrm{kV}$. By using the impulsive noise characteristics, we estimate representative parameters for two impulsive noise models: the Middleton class-A (MCA) and the Bernoulli-Gaussian with memory (BGM).
\end{abstract}

\section{INTRODUCTION}

Impulsive noise in substations remains problematic for Electro-Magnetic Compatibility (EMC) and communication systems such as wireless communications and power line communications (PLC). The emergence of smart grid requires more information about impulsive noise in substations, especially for projects aiming to deploy Intelligent Electronic Devices (IED) for controlling and monitoring substations equipment. Wireless technology would simplify the installation of IEDs within substations, without requiring expensive retrofitting. Moreover, the improving of wireless technology makes it a serious candidate in terms of coverage, delay and throughput.

However, substations host a RF noise, called impulsive noise, that remains a major threat for wireless communications since the power spectrum of the impulses is significant enough to be detected by wireless receivers working in the $700 \mathrm{MHz}-$ 2.5 $\mathrm{GHz}$ band [1], [2]. Installing classic wireless systems within substations could result in data errors, delays and communication interruptions. In substations, impulsive noise is mainly due to electrical partial discharges and Corona effect [2], [3], which are electrical discharges occurring in the insulator of power equipment [3]-[8]. The insulator can be the ambient air around power lines, the transformer oil or the gas within power equipment. Impulsive noise in substations can be considered as the electromagnetic observation of partial discharge and Corona noise, therefore the phenomenon is dependent on the voltage of the equipment where the discharge occurs. The weather, the humidity, solar radiations or even aging of insulators can alter the characteristics of impulsive noise at a precise location. For example, for a same location, we can measure impulsive noise with 10 times more impulses and with a $20 \%$ increasing of amplitude than the day before. Electrical operations, such as switching and circuit-breaking occur within substations and they are also source of impulsive noise.

Substations usually work under different voltage levels and we have observed in our previous works [1], [2] that impulsive noise characteristics change with the voltage level of the equipment. The impulsive noise become more significant in terms of impulse amplitude and repetition rate when the voltage of the power equipment is large.

During our measurement campaign in Hydro-Québec power substations, we have collected electromagnetic noise sequences among different voltage areas $(25 \mathrm{kV}, 230 \mathrm{kV}, 315$ $\mathrm{kV}$ and $735 \mathrm{kV}$ ). Each measurement sequence contains 256 millions of noise samples, which represents a time window of $51.2 \mathrm{~ms}$ with a sampling frequency of 5 Giga-Samples per second $(\mathrm{GS} / \mathrm{s})$. The measurements are performed in the Industrial, Scientific and Medical (ISM) band: $700 \mathrm{MHz}$ $2.5 \mathrm{GHz}$ [2], which contains many existing wireless carrier frequencies.

Different models exist to represent communication channels impaired by impulsive noise and we propose to study two very common models: the Middleton Class-A (MCA) and the Bernoulli-Gaussian with memory (BGM). MCA represents impulsive noise with i.i.d samples, while BGM is a samplecorrelated model that represents impulses with bursts of samples.

In Section II, we present impulsive noise in power substations and we introduce the two models MCA and BGM to represent such a noise. In Section III, we present our 

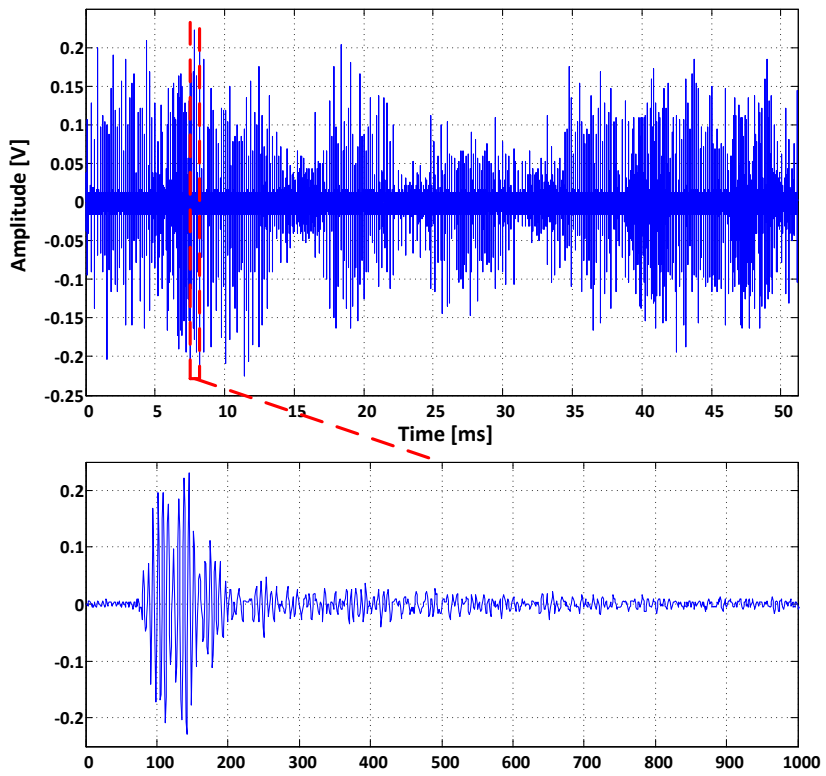

Fig. 1. Impulsive noise in a $735 \mathrm{kV}$ substation.

measurement campaign and the method that we use to classify impulsive noise characteristics, such as the impulse amplitude, the impulse duration, the inter-arrival times (IAT), given the voltage levels of power equipment within the visited substations. Finally, in Section IV, we show the parameters for the two models and for different voltage of substation; we also provide an example of the waveform that the models can generate with our parameters.

\section{IMPULSIVE NOISE AND MODELS}

\section{A. Impulsive noise in power substations}

As we have mentioned in the introduction, impulsive noise is dominant in power substation. This particular RF noise is basically represented by a process that switches from a background noise (usually Gaussian) to another noise, that is represented by an impulse. The samples within an impulse are correlated and they shape the impulse with a damped oscillation waveform (Figure 1). In order to simplify the study of impulsive noise, we introduce, in this paper, the impulsive noise characteristics:

- The impulse sample values are the samples that belong to an impulse;

- The impulse amplitude corresponds to the largest sample, in absolute value, of the samples that belong to an impulse;

- The impulse duration is the time between the first and the last sample of an impulse;

- The repetition rate represents the number of impulse per second. It is directly related to the Inter-Arrival time (IAT), which is the duration between two consecutive impulses.

Impulsive noise can be represented by different models that provide samples with distributions more or less similar to the measured samples. The implementation of correlation between the samples is another aspect that can ensure a better representation of the impulsive noise process. We propose to provide parameters for classic existing impulsive noise models, such as Middleton class-A (MCA) [9] and Bernoulli-Gaussian with memory (BGM) [10], [11].

\section{B. Middleton class-A}

Middleton proposes three models that suit specific communication scenarios, where the impulsive noise is supposed to be series of waves defined by its amplitude and duration $T_{I}$ at the input of the receiver [9].

Moreover the three models assume the following conditions: First, the noise sources are independent. Secondly, there are an infinite number of impulse sources that disturb the communication. Finally, the three models represent impulsive noise at a narrow-band receiver. We call the receiver bandwidth $\Delta_{f_{R}}$.

We present below the three models [12]:

- The Class-A model (MCA) represents impulsive noise when the noise band is narrower than the receiver band. The impulse last much longer than the transient decay period at the input.

$$
T_{I} \Delta_{f_{R}} \gg 1
$$

MCA model is very general and it can be used to represent interferences coming from other communications, electromagnetic emission from machinery, or from power lines.

- The Class-B model, on the contrary, assumes that impulsive noise has a band larger than the receiver bandwidth. The impulse emission duration is negligible compared to the transient decay period.

$$
T_{I} \Delta_{f_{R}} \ll 1
$$

This model characterizes impulsive noise coming from other communications, EM clutter and automobile ignition [12].

- The Class-C is more general and this model assumes the environment to be a mix of class-A and class-B noises. Class- $\mathrm{C}$ has the same criteria in terms of noise-to-receiver band ratio than Class-B model.

$$
T_{I} \Delta_{f_{R}} \ll 1
$$

We prefer to study the class-A model for representing impulsive noise in power substation because it is applicable for interferences coming from power lines and the model requires only three parameters that can be estimated from measurements with different methods [13], [14]. Moreover, the measurement campaigns in [5], [6] show that the impulses can last $1 \mu \mathrm{s}$, which is dominant compared to a $20 \mathrm{MHz}$ Wi-Fi bandwidth, for example.

MCA model considers that the noise sources are distributed according a Poisson distribution and that each source generates 
a Gaussian noise. The pdf $f(x)$ of the sample value at the receiver is

$$
f(x)=\frac{e^{-A}}{\sqrt{2 \pi}} \sum_{m=0}^{\infty} \frac{A^{m}}{m ! \sigma_{m}} \exp \left(-\frac{x^{2}}{2 \sigma_{m}^{2}}\right)
$$

with $\sigma_{m}^{2}=\sigma^{2} \frac{m / A+\Gamma}{1+\Gamma}$. The parameters $\Gamma$ and $A$ characterize respectively the impulses magnitude and their occurrence rate.

- We call $A$ the impulsive index and it characterizes the impulses occurrence [12]. This parameter is equal to

$$
A=\rho \bar{T}
$$

With $\rho$ the number of impulses by second (or repetition rate), and $\bar{T}$ is the mean duration of the impulses. A small impulsive index implies that the impulses are not dominant compared to the background noise. For example, if the impulsive noise has few impulses or/and very short impulses, then $A$ will have a small value. On the other hand, a large impulsive index implies that the impulses are noticeable compared to the background noise, and the more it increases, the more impulsive noise becomes Gaussian.

- We call $\Gamma$ the Gaussian to impulsive noise ratio and it characterizes the power of the impulses [12]. $\Gamma$ is the ratio of the background noise power by the impulse power.

$$
\Gamma=\frac{\sigma_{G}^{2}}{\sigma_{I}^{2}}
$$

The smaller the Gaussian to impulsive noise ratio is and the stronger the impulses are, compared to the background noise [12].

- The parameter $\sigma^{2}$ is the "noise power" (impulse and background noise samples), such as $\sigma^{2}=\sigma_{G}^{2}+\sigma_{I}^{2}$.

- $\sigma_{m}^{2}$ is the variance of the $m^{t h}$ impulsive source. For $m=$ 0 , the model generates the amplitudes of the background noise.

\section{Bernoulli-Gaussian with memory}

The Bernoulli-Gaussian model is certainly the simplest way to represent impulsive noise since it is a mixture of two Gaussian generations of samples ruled by a single Bernoulli probability [11], [15]. The probability density function of the samples $f(x)$ for this model is

$f\left(x \mid \sigma_{0}^{2}, \sigma_{1}^{2}, \lambda\right)=\frac{\lambda}{\sqrt{2 \pi \sigma_{0}^{2}}} \exp \left(-\frac{x^{2}}{2 \sigma_{0}^{2}}\right)+\frac{1-\lambda}{\sqrt{2 \pi \sigma_{1}^{2}}} \exp \left(-\frac{x^{2}}{2 \sigma_{1}^{2}}\right)$

In general cases, one Gaussian distribution characterizes the background noise and the other one, the impulses. The concept of this model is simple because it assumes there is only one source of impulsive noise that generates i.i.d. impulses for a one-sample duration; also the mean of the background noise and the impulses are assumed to be null. According to (7), the model needs only three parameters, which are the background noise variance $\sigma_{0}^{2}$, the impulse variance $\sigma_{1}^{2}$, and the Bernoulli probability $\lambda$ (the probability to be in an impulsive state is $1-\lambda$ ). The model can also be represented by a Markov chain with two states (State 0 for the background noise and State 1 for the impulse in Figure 2), with the following probability matrix:

$$
T=\left(\begin{array}{cc}
\lambda & 1-\lambda \\
\lambda & 1-\lambda
\end{array}\right)
$$

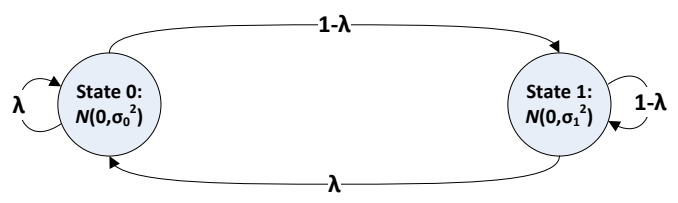

Fig. 2. Bernoulli-Gaussian model (no memory) represented by a Markov chain.

Even if this model does not generate impulsive noise like observations made in power substation, it can represent the impulses occurrence with their sample values. However there is only one source to generate the impulse samples, while there are an infinite number of sources for the MCA model.

In order to make the Bernoulli-Gaussian model close to measurements, we decide to implement the noise process with some memory:

- We implement memory to the Markov chain by selecting different transition probabilities (Figure 3) to generate a "burst of impulses" [11], [16], which is basically a sequence of impulse samples for a specific duration.

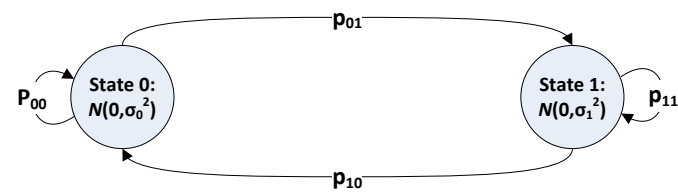

Fig. 3. Bernoulli-Gaussian model with memory.

- We could also add more impulsive noise sources. This modification would require more parameters, such as the impulse variances and the probabilities to transit from the background noise to the impulse. Also, the Bernoulli-Gaussian pdf $f(x)$ should become a mixture of $N$ Gaussian distributions.

$$
f(x)=\sum_{m=0}^{N-1} \frac{\lambda_{m}}{\sqrt{2 \pi \sigma_{m}^{2}}} \exp \left(-\frac{x^{2}}{2 \sigma_{m}^{2}}\right)
$$

with $\sum_{m=0}^{N-1} \lambda_{m}=1$. This possible modification is just a recommendation; we will limit our work to a BGM model with one source of impulse. 
To summarize, the BGM model is a two-state Markov chain, where each state is associated with a zero-mean Gaussian distribution; one state represents the background noise and the other state represents the impulse. The integration of memory by using a Markov chain allows the replication of the impulse duration and impulse amplitude; however, the waveform of the impulse is not a damped oscillation, but a sequence of Gaussian noise with a variance greater than the background noise variance. Such a model can be implemented in receivers that use the memory of the model in order to mitigate the impact of impulsive noise [16].

\section{MEASUREMENT CAMPAIGN: CLASSIFICATION OF IMPULSIVE NOISE INFORMATION}

\section{A. Impulse classification}

With more than 120 noise sequences, we are able to provide all the information required for estimating the parameters of each model and for four voltage levels. We summarize here the method used to classify the impulses from a noise sequence.

The first step for impulse classification is the detection of the impulses. In our previous work [10], we have proposed an impulse detection method (IDM) based on the evaluation of the background noise level. Basically, the method consists in separating the samples above the background noise level, detecting the first and last sample of each impulse and rebuilding each impulse with the samples between the first and the last samples. We refer to our work [10] for more details.

For a specific equipment voltage, we have $N$ noise sequences. For each sequence, called $S e q_{i}$ with $i=1 \ldots N$, we apply the IDM and we classify the samples into two groups: the background noise group (BNG) and the impulse group (IG). We keep the correlation between the impulse samples for IG, since we will need information about the impulse duration and the amplitude.

\section{B. Representative characteristics of impulsive noise}

The calculation of the parameters requires their definition given by the model and the value of the characteristics that we have defined in II.A. We present here the characteristics of impulsive noise that characterized the RF environment for a specific voltage of substation. From the characteristics it will be possible to derive extra information in order to calculate the parameters for the two models.

With the two groups BNG and IG, we calculate the impulsive noise characteristics and present average values than can be used to represent the RF environment for each substation voltage. The average values are calculated using the sample mean and the sample variance. More specifically, the amplitude, the impulse duration and the repetition rate are calculated with the sample mean of the characteristic values classified in the Impulse Group (IG). The background noise variance and the impulse variance are calculated with the sample variance of all the noise samples in BGM and IG respectively. The results are shown in Table I.

Some information about the impulses (Table I) can be used directly to estimate the parameters of the Class-A and BGM.
When studying the impulse duration, we have noticed in our previous works [1], [2] that the impulses last longer when the voltage of the equipment increases; although this correlation is spottable sometimes, the difference between the impulse duration for different voltages is too small to confirm this assumption. The increasing of the impulse power also implies the increasing of the background noise, which makes difficult the reading of the impulse samples when that are contained in the background noise envelope.

\section{PARAMETERS AND MODELS COMPARISON}

We present the method used to calculate the parameters that are the most appropriate to represent impulsive noise within substation for the two models. With the information derived from the impulse classification, we use the parameters definition to calculate their values.

\section{A. Parameters and definition}

In this section, we give the definition and the equation to calculate the parameters for each model. The class-A model uses 3 parameters that we have defined in II.B. The use of the Middleton model for a wide band study can be considered as Narrow band, however the parameter estimation methods proposed so far [13], [14], [17] are not efficient in wide band according to our previous experience [10]. We prefer then to use the definition of the parameters and the average values of the impulses noise characteristics (Table I) to calculate the three parameters $A, \Gamma$ and $\sigma$.

Given the parameter definition ,we can calculate the following parameters:

$$
\begin{gathered}
A=\text { repetiton rate } \times \text { average impulse duration } \\
\Gamma=\frac{\text { background noise variance }}{\text { impulse sample variance }}
\end{gathered}
$$

$$
\sigma^{2}=\text { sampled variance(impulsive noise samples) }
$$

For the implementation of MCA, we chose 5 sources of noises instead of an infinite. Among the 5 sources of noise, one source represents the background, the 4 other sources represent the impulses.

The BGM model requires information about the impulse duration, the repetition rate, the variance of the background noise and the variance of the impulse samples. The impulse duration is represented by the probability to leave state 1 ( $p_{10}$ in Figure 3 ) and the repetition rate is represented by the probability $p_{01}$ to transit from state 0 (background noise) to state 1 (impulse). We have the following equations:

$$
\begin{aligned}
p_{01} & =\frac{\text { number of impulses }}{\text { duration in background noise }} \\
p_{10} & =\frac{1}{\text { average impulse duration }}
\end{aligned}
$$


TABLE II

Class-A PARAMETERS

\begin{tabular}{|c|c|c|c|c|}
\hline & $25 \mathrm{kV}$ & $230 \mathrm{kV}$ & $315 \mathrm{kV}$ & $735 \mathrm{kV}$ \\
\hline$A$ & $1.7 \times 10^{-4}$ & $1.8 \times 10^{-3}$ & $1.7 \times 10^{-4}$ & $2.1 \times 10^{-4}$ \\
$\Gamma$ & $2.9 \times 10^{-2}$ & $2.5 \times 10^{-2}$ & $7.2 \times 10^{-3}$ & $6.1 \times 10^{-3}$ \\
$\sigma^{2}$ & $1.54 \times 10^{-4}$ & $8.4 \times 10^{-4}$ & $4.2 \times 10^{-3}$ & $5.7 \times 10^{-3}$ \\
\hline
\end{tabular}

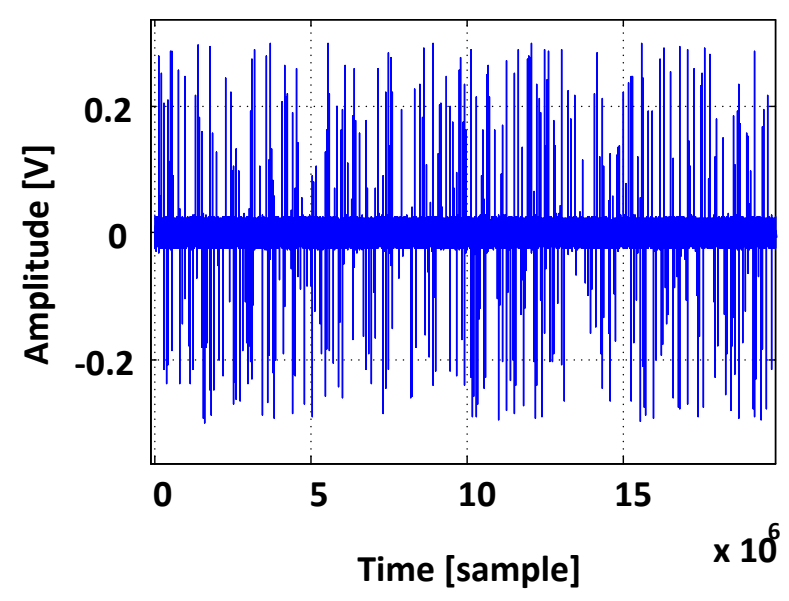

Fig. 4. MCA impulsive noise representing a $735 \mathrm{kV}$ environment.

\section{B. Results}

In order to evaluate the performance of the MCA and BGM models, we generate impulsive noise with the proposed parameters (Tables II and III). The noise sequence length is configured for 20 millions of samples, for both models. We use, for each voltage level (Table II), the parameters calculated from our estimation of impulsive noise characteristics (Table I). We show in Figure 4 an example of noise sequence generated with the MCA model, using our proposed parameters for a $735 \mathrm{kV}$ environment. As we can observe on Figure 4 the noise is composed of i.i.d samples for the MCA model and the impulses samples are generated from 4 Gaussian distributions, which makes this model more realistic for the sample values.

For the BGM model, the parameters are shown in Table III. As for the MCA model, we provide parameters for each voltage level $(25,230,315$ and $735 \mathrm{kV})$ and we show in Figure 5 an example of noise sequence generated by the
TABLE III

BGM PARAMETERS

\begin{tabular}{|c|c|c|c|c|}
\hline & $25 \mathrm{kV}$ & $230 \mathrm{kV}$ & $315 \mathrm{kV}$ & $735 \mathrm{kV}$ \\
\hline \hline$p_{01}$ & $1.3 \times 10^{-6}$ & $2.6 \times 10^{-6}$ & $2.3 \times 10^{-6}$ & $3.3 \times 10^{-6}$ \\
\hline$p_{10}$ & $7.6 \times 10^{-3}$ & 0.013 & 0.014 & 0.0143 \\
\hline$\sigma_{0}^{2}$ & $4 \times 10^{-6}$ & $2 \times 10^{-5}$ & $2.7 \times 10^{-5}$ & $3.5 \times 10^{-5}$ \\
\hline$\sigma_{1}^{2}$ & $1.5 \times 10^{-4}$ & $8.2 \times 10^{-4}$ & $4.1 \times 10^{-3}$ & $5.6 \times 10^{-3}$ \\
\hline
\end{tabular}

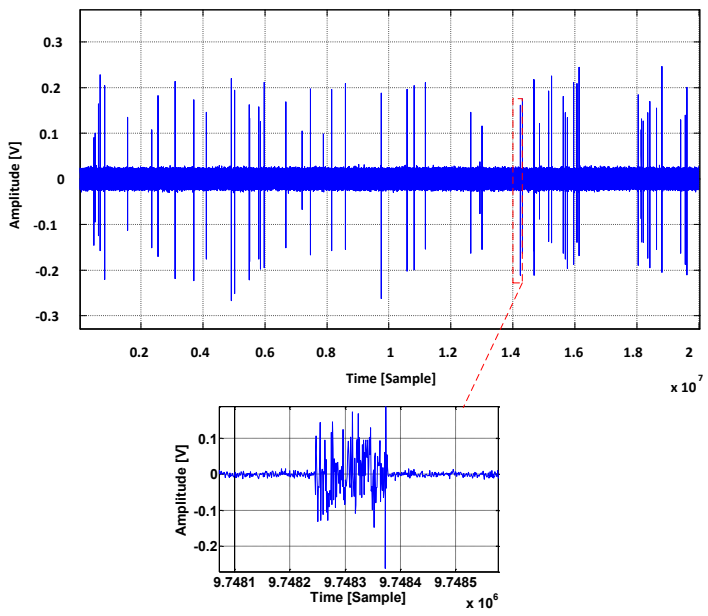

Fig. 5. BGM impulsive noise representing a $735 \mathrm{kV}$ environment.

BGM model with our parameters for the $735 \mathrm{kV}$ environment.

On Figure 5, we observed that the impulses samples are gathered in a burst of impulse, which is more similar with the measurements than the i.i.d samples of MCA, in terms of impulse duration. However, an impulse is represented by one Gaussian distribution, instead of 4 distributions as with MCA model.

\section{CONClusion}

Substation environments generate impulsive noise with characteristics that change with the equipment voltage. We have proposed a method to classify those characteristics for different voltages of substation and we have provided average values as a contribution. We have also calculated parameters that are more suitable for MCA and BGM models in order to represent an RF environment given a specific voltage of substation.

MCA model is more suitable for communication systems assuming i.i.d noise samples, e.g. systems using inter-leaving or

TABLE I

IMPULSE CHARACTERISTICS

\begin{tabular}{|c|c|c|c|c|}
\hline $\begin{array}{l}\text { Voltage area } \\
\text { Impulsive characteristics }\end{array}$ & $25 \mathrm{kV}$ & $230 \mathrm{kV}$ & $315 \mathrm{kV}$ & $735 \mathrm{kV}$ \\
\hline Amplitude (V) & 0.026 & 0.061 & 0.098 & 0.136 \\
Impulse duration (samples) & 132 & 72 & 71 & 70 \\
Repetition rate (impulses/second) & $6.4 \times 10^{3}$ & $1.3 \times 10^{4}$ & $1.2 \times 10^{4}$ & $1.7 \times 10^{4}$ \\
Impulse sample variance & $1.5 \times 10^{-4}$ & $8.2 \times 10^{-4}$ & $4.1 \times 10^{-3}$ & $5.6 \times 10^{-3}$ \\
Background noise variance & $4.2 \times 10^{-6}$ & $2.1 \times 10^{-5}$ & $3 \times 10^{-5}$ & $3.5 \times 10^{-5}$ \\
Total noise variance & $4.4 \times 10^{-6}$ & $2.1 \times 10^{-5}$ & $3.1 \times 10^{-5}$ & $3.9 \times 10^{-5}$ \\
\hline
\end{tabular}


down-sampling. For communications considering the memory of the noise, we recommend to use the BMG model with the proposed parameters, especially for communication receivers that aim to take advantage of the sample correlation to mitigate the impact of impulsive noise.

As future work, we recommend to provide parameters for more complex models, such as partitioned Markov chains and to study the impact of the weather on the characteristics of substation impulsive noise.

\section{ACKNOWLEDGMENT}

This work was supported by Hydro-Québec, the Natural Sciences and Engineering Research Council of Canada and McGill University in the framework of the NSERC/HydroQuébec/McGill Industrial Research Chair in Interactive Information Infrastructure for the Power Grid.

\section{REFERENCES}

[1] F. Sacuto, F. Labeau, and B. Agba, "Evolution of the RF Characteristics of the Impulsive Noise in High Voltage Environment," in IEEE SmartGridComm 2012 Workshop, pp. 686 -691, Oct 2012.

[2] F. Sacuto, F. Labeau, J. Béland, M. Kassouf, S. Morin, S. Poirier, and B. Agba, "Impulsive Noise Measurement in Power Substations for Channel Modeling in ISM Band," in 2012 CIGRE Canada Conference, Sept 2012

[3] R. Bartnikas and E. McMahon, Corona Measurement and Interpretation. ASTM Special Technical Publication, 1995.

[4] M. Hikita, H. Yamashita, T. Hoshino, T. Kato, N. Hayakawa, T. Ueda, and H. Okubo, "Electromagnetic noise spectrum caused by partial discharge in air at high voltage substations," IEEE Transactions on Power Delivery, vol. 13, pp. 434 -439, apr 1998.

[5] C. H. Peck and P. Moore, "A direction-finding technique for wideband impulsive noise source," IEEE Transactions on Electromagnetic Compatibility, vol. 43, pp. 149 -154, may 2001.
[6] I. Portuguas, P. Moore, and I. Glover, "Characterization of radio frequency interference from high voltage electricity supply equipment," in Twelfth International Conference on Antennas and Propagation, vol. 2, pp. 820 - 823 vol.2, march-april 2003.

[7] W. Pakala and V. Chartier, "Radio Noise Measurements on Overhead Power Lines from 2.4 to $800 \mathrm{kV}$," IEEE Transactions on Power Apparatus and Systems, vol. PAS-90, pp. 1155 -1165, may 1971.

[8] W. E. Pakala, E. R. Taylor, and R. T. Harrold, "Radio Noise Measurements on High Voltage Lines from 2.4 to $345 \mathrm{kV}$," in IEEE Electromagnetic Compatibility Symposium Record, pp. 96 -107, july 1968.

[9] D. Middleton, "Non-gaussian noise models in signal processing for telecommunications: new methods an results for class a and class b noise models," IEEE Transactions on Information Theory, vol. 45, pp. 1129 -1149 , may 1999.

[10] F. Sacuto, F. Labeau, and B. Agba, "Wide band time-correlated model for wireless communications under impulsive noise within power substation," IEEE Transactions on Wireless Communications, vol. 13 pp. 1449-1461, March 2014.

[11] H. Ferreira, L. Lampe, J. Newbury, and T. Swart, Power Line Communications: Theory and Applications for Narrowband and Broadband Communications Over Power Lines. John Wiley \& Sons, 2010.

[12] D. Middleton, "Statistical-Physical Models of Electromagnetic Interference," IEEE Transactions on Electromagnetic Compatibility, vol. EMC19, pp. $106-127$, aug 1977.

[13] S. Zabin and H. Poor, "Parameter estimation for Middleton Class A interference processes," IEEE Transactions on Communications, vol. 37, pp. $1042-1051$, oct 1989.

[14] S. Zabin and H. Poor, "Efficient estimation of Class A noise parameters via the EM algorithm," IEEE Transactions on Information Theory, vol. 37, pp. $60-72$, jan 1991.

[15] S. V. Vaseghi, Advanced Digital Signal Processing and Noise Reduction. Wiley, 2008.

[16] D. Fertonani and G. Colavolpe, "On reliable communications over channels impaired by bursty impulse noise," IEEE Transactions on Communications, vol. 57, pp. $2024-2030$, july 2009.

[17] D. Middleton, "Procedures for Determining the Parameters of the FirstOrder Canonical Models of Class A and Class B Electromagnetic Interference," IEEE Transactions on Electromagnetic Compatibility, vol. EMC-21, aug 1979. 